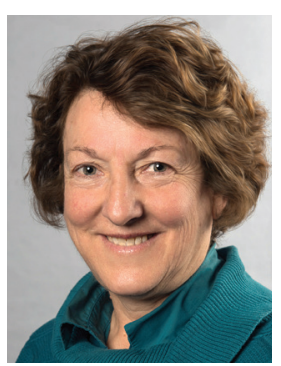

Margaret Hamilton

RMIT University

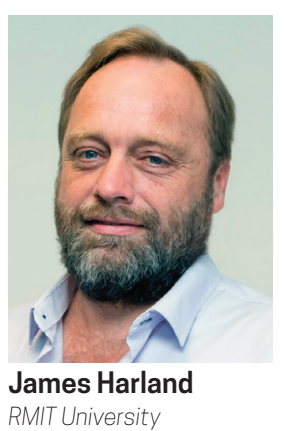

RMIT University

\section{EDITORS' MESSACE}

\title{
Welcome to 2022 !
}

We start the year with an excellent interview of Mark Guzdial. In our expert series of interviews with prominent propagators, David Bunde, Zack Butler, Christopher L. Hovey and Cynthia Taylor offer the latest interview with some wide-ranging and very interesting questions. Mark Guzdial comes up with thought-provoking answers ranging from what liberal arts professionals might do with programming and Computer Science in general to 'Computing for Discovery' and 'Computing for Justice,' as well as considerations of cognitive load and the importance of context. However, the most important answers, in our view, consider the overall need for more computing education research, how it is conducted and what it can tell us. It would be great if every reader would consider applying the messages in his responses to their own context in teaching and how it could work so well for them.

Orit Hazzan and Koby Mike explain how machine learning can be taught in a high school setting using only the KNN algorithm. In their interesting offering, the authors provide many examples of how the KNN algorithm can be used to demonstrate machine learning techniques discussed in the classroom. The authors also suggest other suitable algorithms that could be applied based on their analysis of computational, cognitive, and pedagogical perspectives, depending on the audience and teaching objectives. In this case, however, the examples are provided for high school students of a data science course, requiring middle school mathematics. The article provides interesting food for thought.

David Ginat's column on the 'Domino Line' is a most intriguing extension of his previous column requiring recursion. His last column took the idea of a stairway and devised recursive ideas for traversing the stairway. He now extends this idea by considering a domino as a tuple, to derive an n-duple kind of stairway! The solution he discusses seeks an illuminating recursive perspective, of decomposing the task cases into suitable disjoint subsets, and re-combining them in a most elegant and concise manner.

In his column, Curricula Syncopations, Henry Walker discusses identifying student learning outcomes early. He provides some guiding principles which could be applied as we are organising our courses for Semester 1. The four main principles discussed have wide ranging payoffs, which are also worth considering as is the need to review courses early, often, and regularly.

Scott Weiss provides his second Back Page Puzzle on Word Circuits. Whether it be about puzzles, or interviews, or articles, or our regular columns, we hope you all find something intriguing in this March edition, and we are always interested in your feedback.

We have included the Best Paper from ICER 2021, the ACM International Computing Education Research conference and two of the papers that received Honorable Mentions. These are well worth reading as they highlight new directions in perspectives, as well as enhance our understanding of the evaluation of assessments and novice misconceptions.

We selected the cover image to depict the kind of 'hybrid' or 'integrated' type of classrooms with which we are becoming all too familiar. We look forward to your research, ideas, and evidence on what works well in these kinds of teaching and learning environments. Please consider offering your thoughts for inclusion in an issue of Inroads.

\section{Margaret Hamilton and James Harland}

Editors-in-Chief 